\title{
Les territoires du football en Tunisie (1906-2015)
}

Territories of soccer in Tunisia (1906-2015)

\section{Ali Langar, Myriam Baron et Claude Grasland}

\section{OpenEdition}

\section{Journals}

Édition électronique

URL : http://journals.openedition.org/echogeo/16209

DOI : 10.4000/echogeo.16209

ISSN : 1963-1197

Éditeur

Pôle de recherche pour l'organisation et la diffusion de l'information géographique (CNRS UMR 8586)

\section{Référence électronique}

Ali Langar, Myriam Baron et Claude Grasland, « Les territoires du football en Tunisie (1906-2015) »,

EchoGéo [En ligne], Sur le Vif, mis en ligne le 11 décembre 2018, consulté le 19 avril 2019. URL : http:// journals.openedition.org/echogeo/16209; DOI : 10.4000/echogeo.16209

Ce document a été généré automatiquement le 19 avril 2019

\section{(c) (i) (3)}

EchoGéo est mis à disposition selon les termes de la licence Creative Commons Attribution - Pas d'Utilisation Commerciale - Pas de Modification 4.0 International 


\section{Les territoires du football en Tunisie (1906-2015)}

Territories of soccer in Tunisia (1906-2015)

Ali Langar, Myriam Baron et Claude Grasland

\section{Introduction : enjeux géographiques et politiques du football}

\section{Football et politique en Tunisie}

1 Si l'on se réfère à une partie des évènements qui ont conduit à la révolution dite "de jasmin » en Tunisie marquée par le départ de Zine El Abidine Ben Ali en janvier 2011, notamment les manifestations dans les stades de football, on serait tenté de dire que l'histoire du football se confond avec l'histoire de la Tunisie. C'est ce qui ressort des propos d'un « ultra » du Club Africain de Tunis :

Dans le virage, c'était le bordel. Il y avait des bagarres avec la police, des fumigènes à chaque match malgré les sept contrôles de police à l'entrée des stades, des chants ouvertement anti-gouvernement. C'est en cela que les ultras tunisiens ont joué un rôle dans la révolution, parce que c'était une première en Tunisie de voir des gens résister à la police et critiquer ouvertement le gouvernement. Le seul endroit où la police n'était plus toute puissante, c'était dans le virage. Cela a eu un réel impact sur la population, surtout sur les jeunes, qui en représentent une grosse partie. C'est quand les gens ont vu les ultras aller chaque samedi au stade avec l'objectif d'en découdre avec la police, et que celle-ci était impuissante, qu'une sorte de fatalité a disparu. ${ }^{1}$

2 Ces manifestations semblaient faire écho à celles qui avaient eu lieu avant l'indépendance du pays en 1956. L'existence d'équipes de football sur le territoire tunisien renvoie aussi à la manière dont ce sport s'est diffusé pendant le protectorat français à partir de 1881, selon des proximités spatiales, sociales et économiques mais aussi, de manière moins centrale, en fonction de la hiérarchie urbaine. De telles dynamiques ont été également étudiées dans d'autres contextes, par exemple en France (Ravenel, 1998a, 1998b, 2004) en 
adoptant une approche spatiale de la diffusion des innovations (Saint-Julien, 1982,1985 ; Sanders, 2001). Ces proximités et hiérarchies renvoient en effet à des inégalités spatiales et territoriales, qui parfois se sont maintenues parfois se sont renforcées depuis l'indépendance du pays (Belhedi, 1994, 1999 ; Gana, 2011 ; Signoles, 1978). Parmi ces inégalités, il convient de rappeler l'opposition révélée par la révolution de Jasmin entre un littoral concentrant les principales richesses et les principales agglomérations et une zone intérieure plutôt démunie et rurale sans véritable pôle de développement (Belhedi, 2015 ; Bennasr et al., 2015).

\section{La répartition « égalitaire » des clubs de D1 après la révolution de Jasmin}

Pour un observateur naif, la distribution des clubs de Ligue 1 tunisiens dans les années qui suivent la révolution de Jasmin pourrait apparaitre relativement harmonieuse, si l'on entend par là une couverture régulière du territoire (pas de zones trop éloignées d'un club de Ligue 1) et une adéquation au poids démographique (proportionnalité entre le nombre de clubs et le nombre d'habitants). Comme le montre L'illustration 1, chacune des 12 ligues régionales qui organisent le football tunisien a eu au moins une fois un représentant dans le sommet du championnat entre 2012 et 2015, même si ce fut pour une seule année. Il existe certes une très forte concentration des clubs de Ligue 1 présent durant l'ensemble des saisons dans le Grand Tunis et, à un moindre degré, dans les grandes agglomérations littorales (Sousse, Bizerte, Sfax, Gabès). Mais on trouve également des clubs affichant une présence pérenne dans le Sud (Gabès, Zarzis, Ben Guerdane) et dans les régions de l'intérieur (Béja, Metlaoui, Gafsa). Le calcul d'un potentiel de présence des clubs de Ligue 1 dans un rayon de $50 \mathrm{~km}$ dessine une configuration finalement assez proche de l'image du potentiel de population dans un même rayon en 2014. 


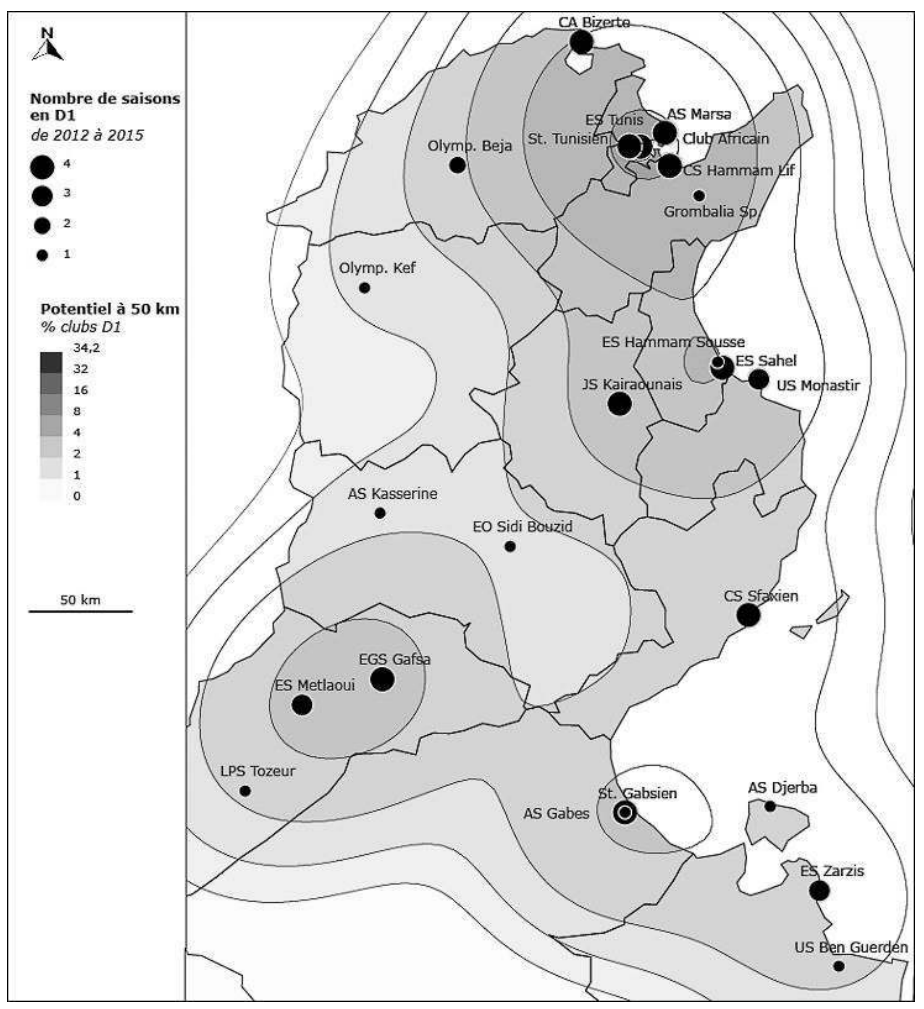

Source: A. Langar, travaux de doctorat en cours.

4 La question qui se pose alors est de savoir si la configuration observée en 2012-2015 constitue une rupture avec les tendances antérieures ou si cette configuration "égalitaire» n'est que le résultat d'une diffusion s'inscrivant dans un processus historique de longue durée. Est-elle une parenthèse temporaire ou définit-elle un équilibre durable? Résulte-t-elle d'une action délibérée du pouvoir politique et des autorités sportives, notamment à partir des découpages territoriaux utilisés pour organiser l'ensemble des compétitions? Ou bien est-elle l'effet du jeu d'autres facteurs : économiques, politiques ou sociaux?

\section{Trois axes d'explication}

5 On ne peut bien évidemment pas comprendre la distribution actuelle des clubs de football en Tunisie sans l'inscrire dans une perspective spatiale et temporelle multi scalaire (selon l'approche de Gaubert, 2016 étudiant l'implantation différenciée, hiérarchisée et segmentée des pratiques de football dans l'espace géographique global puis français). Dans un premier temps, nous caractériserons donc les manières dont les premières équipes de football ont été implantées sur le territoire du protectorat tunisien. Dans un deuxième temps, à partir d'une base de données rendant compte des trajectoires des équipes de football dans les principaux championnats tunisiens de 1956 à 2016, nous présenterons les inégalités nationales à partir de la distribution des clubs de Ligue 1 sur l'ensemble du territoire tunisien et ses dynamiques depuis l'indépendance. Nous nous centrerons également sur les inégalités «régionales» à travers l'analyse de la distribution des clubs de Ligue 2 et leur organisation en zones aux limites fluctuantes, 
avant de confronter, dans une dernière partie, les maillages des Ligues de football avec les maillages administratifs.

\section{Les origines coloniales de la carte contemporaine du football en Tunisie}

6 L'objectif de cette première partie est de comprendre dans quelle mesure la diffusion initiale du football en Tunisie a pu influer sur les inégalités actuelles de répartition de la pratique de haut niveau. Après avoir souligné l'importance de la métropole tunisienne comme premier foyer d'accueil, on montrera que des formes spécifiques de diffusion se sont mises en place à l'époque coloniale, notamment en lien avec des activités économiques (arsenal de Ferryville, bassin minier de Gafsa)

\section{Les Tunis du football}

7 Si le football est arrivé avec l'instauration du protectorat français en Tunisie en 1881, ce n'est qu'en 1906 qu'est créée la première équipe de football : le Racing Club de Tunis (RCT). Il faut encore attendre quatre années pour que soit organisée la première compétition officielle de football sous le nom de championnat de première série et à laquelle participaient 6 clubs, tous localisés dans la capitale, Tunis (illustration 2). Il s'agissait d'un championnat organisé et joué essentiellement par des Français même si, la même année, les Juifs tunisois créent leur premier club. Des effets de regroupement affinitaire par quartier ou groupes sociaux étaient présents à l'intérieur d'un football qui était alors un phénomène essentiellement urbain (donc avec des fonctions particulières comme l'étudie Grosjean, 2004 dans la France contemporaine).

Il faut enfin attendre les lendemains de la première Guerre mondiale pour que soit créée en 1921 la Ligue Tunisienne du Football (LTF), affiliée à la Fédération Française du Football (FFF). Cette Ligue tunisienne regroupait alors 43 équipes, qui évoluaient en Divisions I et II mais aussi Promotions I et II, aussi bien à Tunis que dans les districts du Centre-Sud et du Nord. 


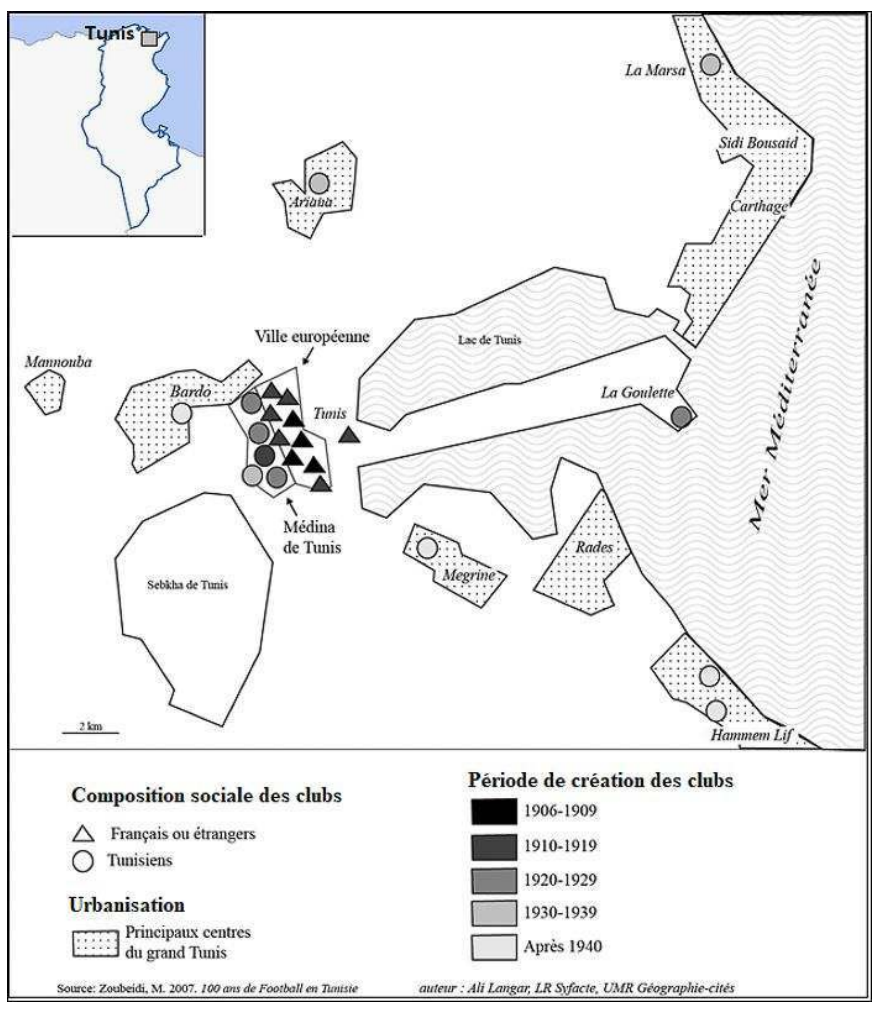

Source : M. Zoubeidi (2007). Auteur : A. Langar.

Même si le football a été perçu comme un révélateur de la présence des Européens sur le territoire du protectorat français, la pratique de ce sport se diffuse jusqu'à l'indépendance en 1956 dans tous les groupes de populations: Français bien entendu, Italiens et Maltais, mais aussi les populations autochtones de juifs et de musulmans. Il devient de loin le premier sport pratiqué par les Tunisiens, comme le révèlent les nombres de licenciés au début des années 1960 : avec environ 4590 licenciés, le football concentrait $39 \%$ de l'ensemble des licenciés dans toutes les fédérations sportives du pays, loin devant l'athlétisme et le basket-ball qui représentaient chacune environ $10 \%$ de ce même ensemble (UNESCO, 1960). Encore aujourd'hui, c'est le sport qui compte le plus de licenciés : 56 \% des quelques 55640 licenciés des cinq premières fédérations de sports collectifs tunisiens.

\section{Ferryville et Gafsa, quand le football s'adosse à l'industrie}

Outre Tunis et ses quartiers, principaux foyers d'apparition et de diffusion du football en Tunisie, d'autres villes parfois proches de la capitale, caractérisées par une spécificité industrielle ont abrité très tôt des équipes de football. Parmi ces villes, Ferryville - actuelle Menzel Bourguiba située à $60 \mathrm{~km}$ au Nord-Ouest de Tunis - présente un exemple original de diffusion spatiale de voisinage, fondée sur des réseaux sociaux et économiques. Il s'agit d'une ville nouvelle créée par les Français dès 1897 sur un site stratégique entre les lacs d'Ichkeul et de Bizerte, ceci pour en faire un arsenal. En 1900, trois ans après sa fondation, Ferryville compte déjà 5000 habitants, dont 1800 Tunisiens, 1000 Français et 2200 étrangers (Maltais, Espagnols et surtout Italiens de Sicile). 
Ferryville est l'une des premières villes de Tunisie à avoir adopté le football en dehors de Tunis. Le Stade ferryvillois voit ainsi le jour en 1909, puis c'est au tour du Sporting Club ferryvillois en 1912. Au-delà, la diffusion du football dans cette ville nouvelle présente de nombreuses similitudes avec celle de Tunis. En effet, avant la première guerre mondiale, le football n'est pratiqué que par des Ferryvillois d'origine européenne. Après la première guerre mondiale, les Tunisiens commencent à être intégrés dans les équipes. Cette période d'entre deux guerres mondiales est surtout marquée par l'affirmation d'un sentiment nationaliste tunisien, qui s'exprime dans des mouvements politiques, culturels et syndicaux. Le football participe de ce mouvement avec la création du Stade Africain de Ferryville, composé uniquement de Tunisiens. C'est également au cours de cette période que trois stades de football sont construits à Ferryville : « Rien d'étonnant donc à cela et c'est, tout naturellement, l'Arsenal, principal employeur à Ferryville, qui est aussi le grand parrain et pourvoyeur de moyens pour le sport. L'Arsenal mais également la Marine. Évidemment à Ferryville plus qu'ailleurs l'un est presque indissociable de l'autre. $»^{2}$ Après 1956, le Stade Africain de Ferryville va changer de nom pour s'appeler le Stade Africain de Menzel Bourguiba et rester la seule équipe de football dans la ville. L'exemple de Ferryville souligne l'influence décisive de l'industrie et de l'armée dans la diffusion du football. Parallèlement aucune dimension ethnique et communautaire ne transparaît dans la dénomination des équipes puisque la plupart d'entre elles ont pris le nom de la ville, ce qui n'était pas le cas à Tunis où les appellations communautaires étaient nombreuses. Le cas de Ferryville est évidemment particulier. Il ne permet pas de comprendre comment le football s'est diffusé sur de plus longues distances, notamment dans le Sud du pays. La diffusion est-elle plutôt portée par la localisation des industries coloniales ou par la proximité de grandes villes ? Pour apporter des éléments de réponse à cette question, nous nous appuyons sur l'exemple de la zone minière de Gafsa au Sud-Ouest du pays.

11 Même si les Français se sont concentrés essentiellement à Tunis et dans les grandes villes littorales, avec le temps et, surtout, avec la découverte de ressources naturelles à l'intérieur du pays, les colons se sont installés dans des parties du pays plus éloignées des espaces côtiers. Parmi les zones riches en ressources naturelles figure la région de Gafsa avec ses phosphates utilisés comme engrais dans l'agriculture. Les premiers gisements tunisiens sont découverts dès 1885. La Compagnie du Chemin de Fer et des Phosphates de Gafsa, qui reçoit en concession les mines de Metlaoui et Redeyef, est créée dès 1896. Enfin, pour assurer le transport du phosphate vers le littoral, est créée en 1913 la voie ferrée Sfax-Gafsa. On assiste alors à un recrutement international important : Français (cadres), Italiens, Kabyles et Souafas (Oued Souf en Algérie), Tripolitains et Marocains (ouvriers). La région de Gafsa a ainsi concentré la plus forte proportion d'étrangers en Tunisie en 1926 : $17 \%$, contre 3,4 \% pour la moyenne nationale. C'est encore une fois la présence de nombreux colons, surtout français et européens, qui a contribué à l'adoption du football dans cette région. Même si les premières pratiques de ce sport sont en effet mentionnées avant la première guerre mondiale, il faut attendre les années 1920-1925 pour que soit créé officiellement le club de Com-Phos par des ouvriers et des cadres de la Compagnie (Com) des phosphates (Phos) de Gafsa. L'exemple du bassin minier de Gafsa souligne le rôle de l'industrie coloniale dans la diffusion du football, comme à Ferryville. Il met également en évidence des effets de diffusion de proximité entre les villes du bassin.

12 Jusqu'à l'indépendance, la diffusion spatiale $d u$ football en Tunisie est ainsi multifactorielle et renvoie au moins à deux modèles géographiques différents. Le premier correspond à un modèle intra-urbain de diffusion selon le principe de proximité, comme 
à Tunis : la pratique du football s'est diffusée des quartiers européens vers la Médina, selon un processus à la fois spatial et social, le contact et le voisinage de différentes composantes de la population ayant eu une influence directe sur la propagation de ce sport (illustration 1) (processus, étudiés par Lefebvre et al., 2013 en terme de territorialité au sein de la ville). Le second est un modèle inter-urbain de diffusion lié à l'influence économique des entreprises coloniales avec les mises en réseau qu'elles induisent, comme dans le cas de Ferryville ou la zone minière de Gafsa. Ces formes spécifiques de diffusion du football en Tunisie se combinent avec des règles plus générales observables dans tous les pays du monde (Augustin, 1989 ; Ravenel, 1996 ; Dietschy, 2010 ; Gomez, 2014).

\section{Le football des première et deuxième divisions de 1955 à 2015, une lente et difficile déconcentration}

13 Les implantations des principaux clubs de football avant l'indépendance opposaient surtout le Nord et le Sud, en relation avec la structure du protectorat : administrations civiles au Nord et militaires au Sud (Signoles, 1978). Ces implantations ne suivaient qu'imparfaitement la hiérarchie urbaine car elles étaient liées initialement à des logiques intriquées de réseaux économiques, de proximités sociales et de domination politique. La situation change radicalement avec l'indépendance, si l'on observe la distribution spatiale des clubs de Ligue 1 (L1). Extraordinairement concentré à Tunis et dans les métropoles du littoral à la fin des années 1950, le football de première division ne va que très progressivement se diffuser vers l'intérieur du pays ou vers les centres urbains de rang inférieur. C'est ce que montre la base de données inédite que nous avons constituée sur les principales équipes de football en Tunisie avec leur localisation géographique ${ }^{3} .$.

\section{La très lente déconcentration du football de première division}

La distribution des clubs de L1 par grandes périodes met en évidence un mouvement très lent mais continu de déconcentration des clubs de l'élite du football (illustration 3).

Les années qui précèdent et suivent l'indépendance (1955-1969) correspondent à une concentration presqu'absolue du championnat de L1 sur le littoral, de Bizerte à Gabès, avec un centre de gravité situé entre Tunis et Sousse. L'unique exception à cette règle est l'Olympique du Kef (OK), qui est l'un des plus anciens clubs de football tunisien remontant à 1922. Bien que localisé sur le littoral, le Stade de Gabès (SG) occupe une position elle aussi très marginale, étant le seul représentant du Sud tunisien. La période de retour à l'économie de marché, sous Bourguiba (1970-1986), se traduit par une dissémination progressive des clubs de Ligue 1 autour du cœur initial ainsi qu'autour des têtes de ponts observées dans les régions du Sud et de l'intérieur. Les ellipses de dispersion tendent ainsi à s'élargir. Cependant, malgré un très léger déplacement du centre de gravité vers le Sud, c'est toujours le long de l'axe Bizerte-Tunis-Sousse-Sfax que l'on trouve l'écrasante majorité des clubs. Pendant la période de libéralisation économique sous Ben Ali (1987-2011) la professionnalisation croissante du football après 1994 et les nouvelles options d'aménagement du territoire au profit des métropoles et du littoral auraient pu provoquer un retour à la concentration. Mais ce n'est pas ce que l'on observe. Bien au contraire, on assiste à un déplacement net du centre de gravité vers le Sud et un élargissement des ellipses de dispersion vers les régions intérieures. 
La révolution de Jasmin (2011-2015) n'a donc fait qu'accélérer une tendance déjà à l'œuvre dans les dernières années du règne de Ben Ali. Elle conduit surtout très clairement à un renforcement net du rééquilibrage au Sud puisque le centre de gravité du championnat de Ligue 1 se situe désormais à Kairouan alors qu'il était localisé entre Sousse et Tunis au moment de l'indépendance. On notera par ailleurs la remarquable simplification de la carte des clubs à l'intérieur du Grand Tunis au cours du temps (illustration 4). Dès 1987, la compétition dans la métropole se réduit pour l'essentiel à cinq clubs dont la présence en Ligue 1 est pérenne et qui sont localisés à l'Ouest, au Nord et au Sud de l'agglomération.

Illustration 3 - La diffusion spatiale des clubs de football de D1 en Tunisie (1955-2011)

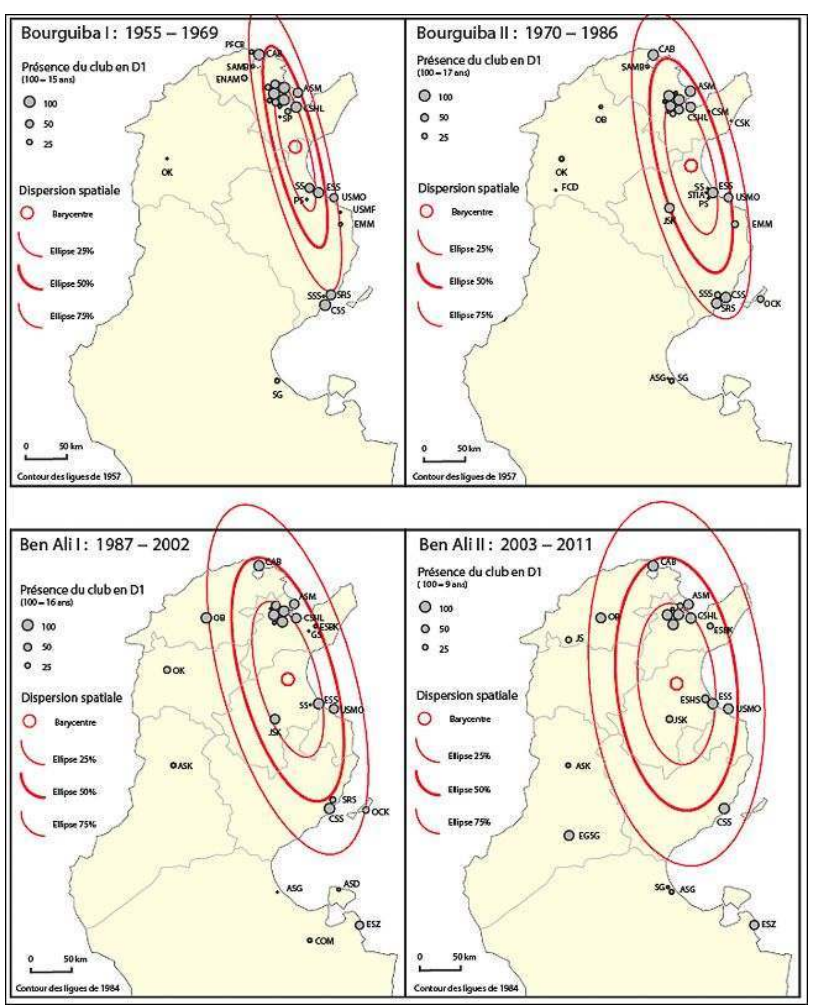

Pour faciliter la comparaison entre des périodes de durées différentes, nous avons représenté les clubs présents avec une taille indiquant le nombre d'années passées en L1. À chaque période, la taille maximale du cercle est identique et renvoie à une présence continue pendant toutes les années de championnat. Leur sont superposés le centre de gravité des clubs (point rouge) et trois ellipses théoriques de dispersion indiquant les zones où se trouvent respectivement le quart, la moitié et les trois-quarts des clubs de L1. Cette représentation s'inspire de celles utilisées dans d'autres contextes et pour d'autres dynamiques géographiques (Béguin, 1979 ; Drevon et al., 2014 ; Keïta, 2018). Pour la liste des sigles se reporter à l'Annexe.

Sources : M. Kilani (2009) pour les données antérieures à 2008 et travaux de A. Langar en cours pour la période suivante et l'harmonisation. 
Illustration 4 - La diffusion spatiale des clubs de football de D1 en Tunisie (1955-2011). Zoom sur la région de Tunis

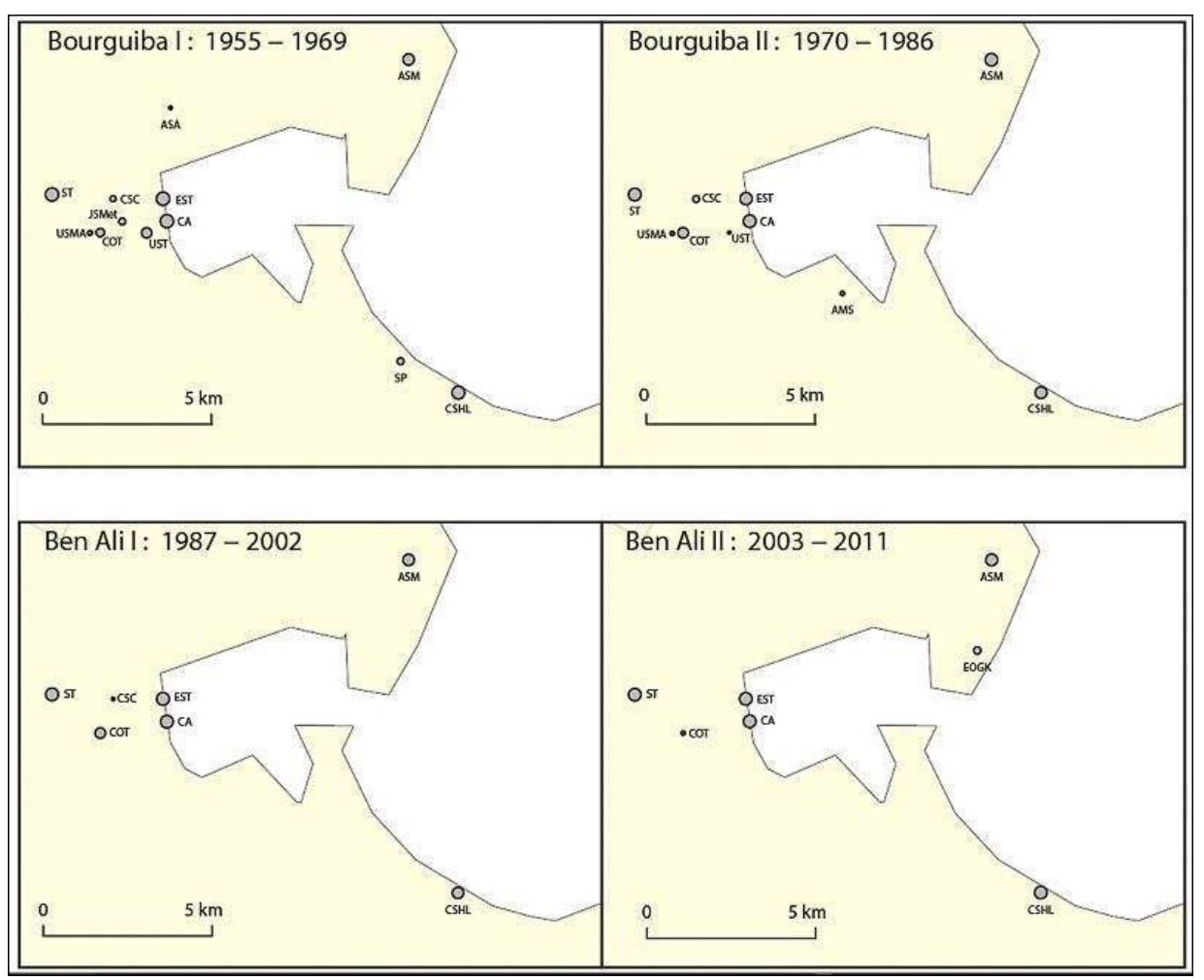

Sources : M. Kilani (2009) pour les données antérieures à 2008 et travaux de A. Langar en cours pour la période suivante et l'harmonisation.

\section{La seconde division comme facteur de rééquilibrage territorial et de promotion}

On ne peut toutefois pas comprendre les dynamiques territoriales du football en Tunisie sans faire intervenir le rôle crucial de la seconde division qui sert tour à tour d'antichambre pour les clubs en cours de promotion et de purgatoire pour ceux qui connaissent une relégation. Leur dispersion Leur répartition spatiale au cours des différentes périodes entre 1955 et 2011 est très sensiblement différente, avec dès l'indépendance, une dispersion beaucoup plus forte en raison de la présence de clubs périphériques situés loin du littoral et hérités pour certains de la période coloniale comme on l'a vu au sujet du bassin minier de Gafsa (illustration 5). 
Illustration 5 - La diffusion spatiale des clubs de football de D2 en Tunisie (1955-2011)
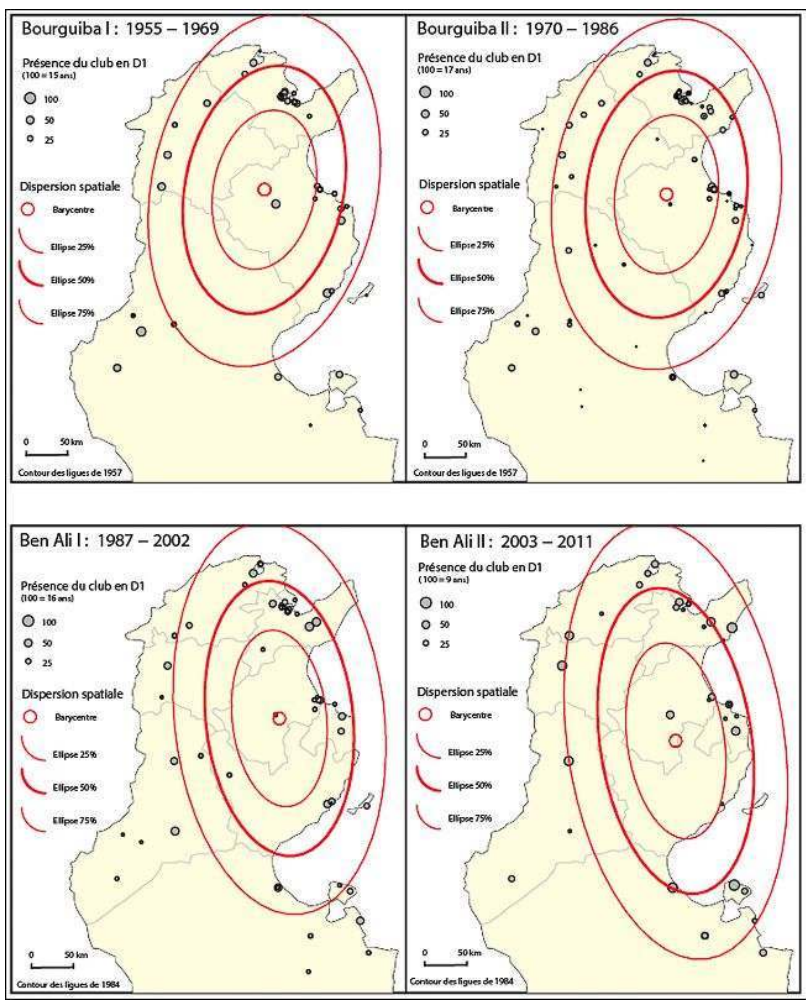

Sources : M. Kilani (2009) pour les données antérieures à 2008 et travaux de A. Langar en cours pour la période suivante et l'harmonisation.

18 Cette distribution égalitaire tend à se maintenir au cours du temps tout en s'accompagnant d'un glissement progressif du championnat de Ligue 2 vers le Sud. Si l'on considère la dynamique de l'ensemble des clubs de $1^{\text {ère }}$ et $2^{\mathrm{e}}$ division dans la durée, on peut mettre en évidence un double mouvement de déconcentration et de diffusion du football vers le Sud-Ouest (illustration6). Le déplacement du centre de gravité et l'accroissement de la distance-type ${ }^{4}$ de près de 61\% durant la période 1955-2015 (le rayon passant de 105 à $168 \mathrm{~km}$ ) en est l'illustration. 
Illustration 6 - Évolution de la dispersion spatiale des clubs de D1 et D2

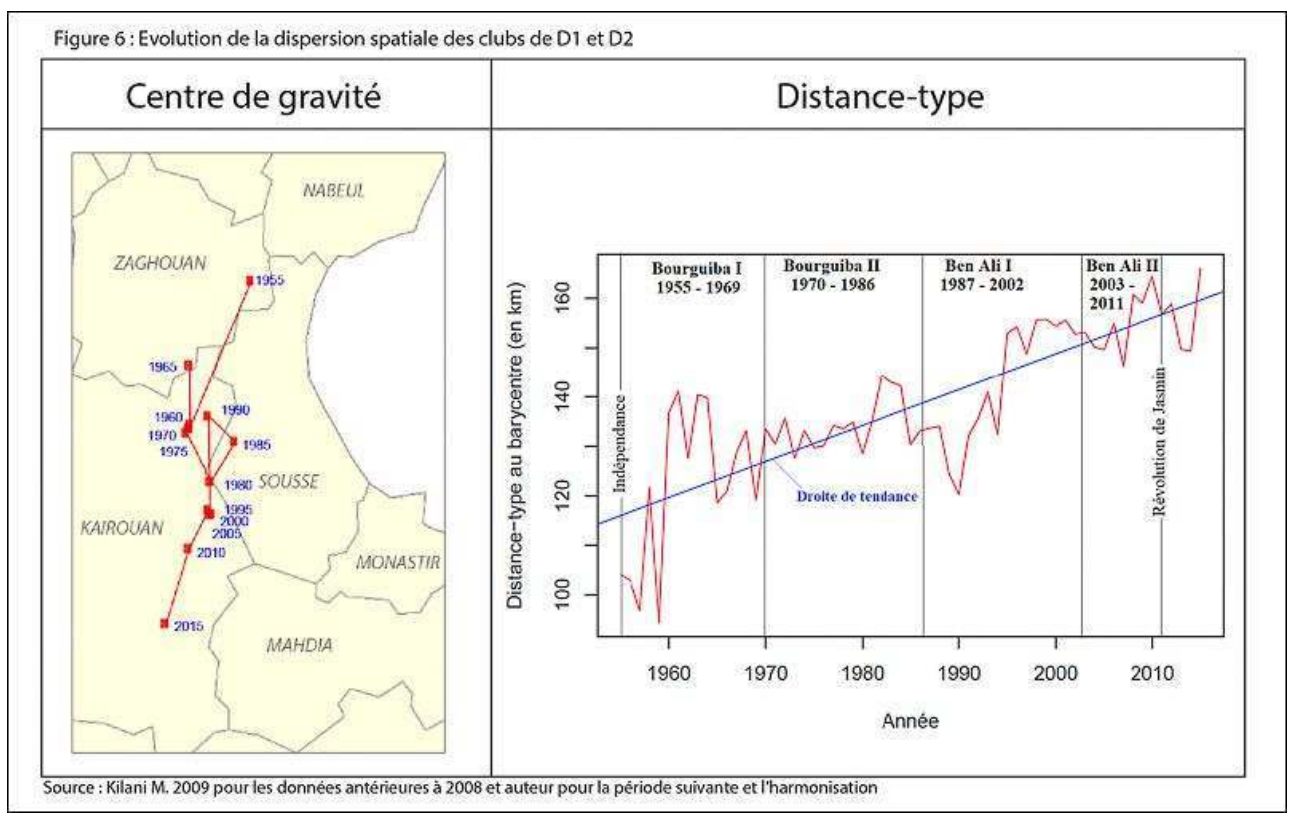

Sources : M. Kilani (2009) pour les données antérieures à 2008 et travaux de A. Langar en cours pour la période suivante et l'harmonisation.

\section{La circulation des clubs entre $1^{\mathrm{èr}}$ et $2^{\mathrm{e}}$ divisions}

Derrière ces tendances statistiques se dissimule une somme d'aventures individuelles de clubs qui naviguent au cours de la période entre les deux premières divisions avec des fortunes variables, voire disparaissent. Quelques exemples permettent de mieux comprendre les modalités concrètes de cette diffusion progressive du football vers l'intérieur, le Sud et les villes de rangs inférieurs (illustration 7). 


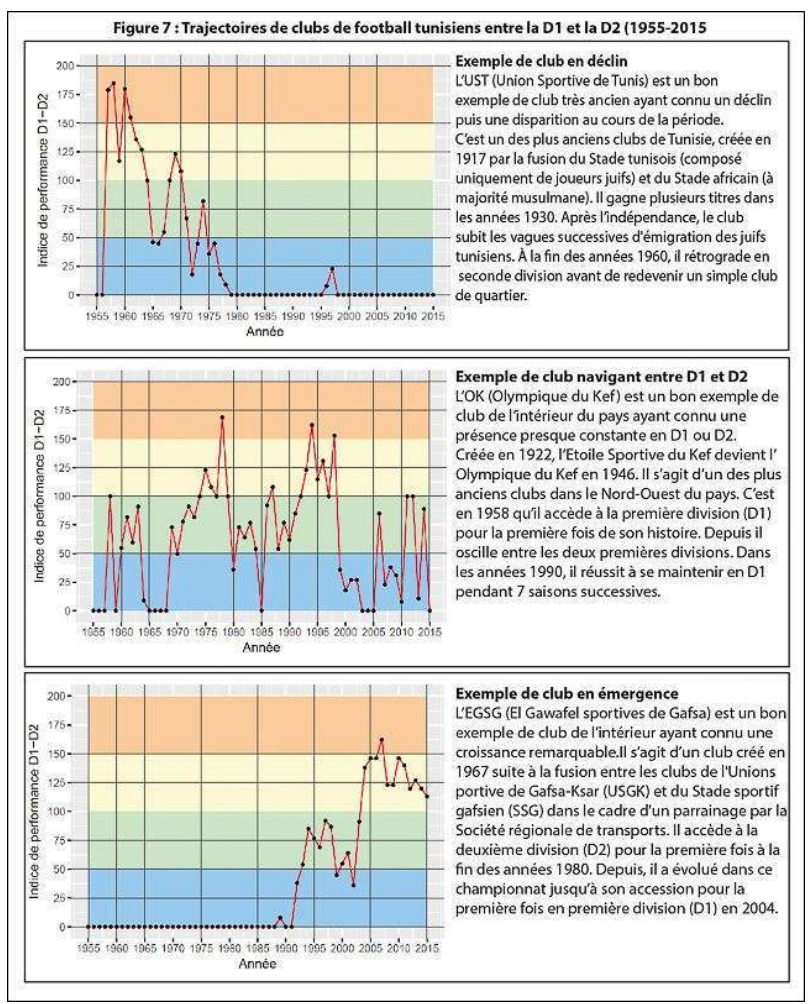

Pour rendre compte des évolutions des clubs sur l'ensemble du continuum constitué par les deux premières divisions, un indice variant entre 0 et 200 a été constitué, tel que 0 correspond à la dernière place de D2, 100 à la première place de D2 ou à la dernière place de D1 et 200 à la première place de D1. Cet indice permet d'éliminer les biais liés au changement du nombre de clubs chaque année et de suivre leurs performances respectives.

Sources : M. Kilani (2009) pour les données antérieures à 2008 et travaux de A. Langar en cours pour la période suivante et l'harmonisation.

Ces mécanismes de rééquilibrage ne doivent cependant pas dissimuler le fait que les grandes métropoles côtières se caractérisent par l'existence de quelques très grands clubs, qui sont présents de façon quasi permanente en D1 et qui y occupent les premières places à tour de rôle. Il est finalement très rare de voir d'autres clubs de l'intérieur ou du Sud occuper la tête du championnat. Il y a sans doute eu une redistribution spatiale des clubs présents en Division ou Ligue 1, mais sans que cela remette en cause la domination des métropoles littorales. Un tableau des clubs ayant remporté le championnat de première division entre 1955 et 2015 le montre (Tableau 1) $)^{5}$.

Tableau 1 - Liste des clubs ayant remporté le championnat de D1 entre 1955 et 2015

\begin{tabular}{|l|l|l|l|l|l|l|l|l|}
\hline Code & Nom & $\begin{array}{l}1^{\mathrm{e}} \\
\text { place }\end{array}$ & $\begin{array}{l}2^{\mathrm{e}} \\
\text { place }\end{array}$ & $\begin{array}{l}3^{\mathrm{e}} \\
\text { place }\end{array}$ & $\begin{array}{l}4^{\mathrm{e}} \\
\text { place }\end{array}$ & $\begin{array}{l}5^{\mathrm{e}} \\
\text { place }\end{array}$ & $\begin{array}{l}6^{\mathrm{e}} \text { place } \\
\text { et plus }\end{array}$ & $\begin{array}{l}\text { Total des } \\
\text { présences en D1 }\end{array}$ \\
\hline EST & $\begin{array}{l}\text { Espérance Sportive } \\
\text { de Tunis }\end{array}$ & 25 & 12 & 10 & 3 & 2 & 8 & 60 \\
\hline CA & Club Africain & 11 & 20 & 12 & 7 & 5 & 6 & 61 \\
\hline
\end{tabular}




\begin{tabular}{|c|c|c|c|c|c|c|c|c|}
\hline ESS & $\begin{array}{l}\text { Etoile Sportive de } \\
\text { Sousse }\end{array}$ & 9 & 17 & 17 & 6 & 5 & 5 & 59 \\
\hline CSS & $\begin{array}{ll}\text { Club } & \text { Sportif } \\
\text { Sfaxien } & \end{array}$ & 8 & 2 & 7 & 14 & 7 & 23 & 61 \\
\hline ST & Stade Tunisien & 4 & 3 & 6 & 9 & 6 & 33 & 61 \\
\hline $\mathrm{CAB}$ & $\begin{array}{l}\text { Club Athlétique } \\
\text { Bizertin }\end{array}$ & 1 & 2 & 1 & 5 & 8 & 42 & 59 \\
\hline CSHL & $\begin{array}{l}\text { Club Sportif } \\
\text { Hammam-Lif }\end{array}$ & 1 & 0 & 0 & 4 & 3 & 47 & 55 \\
\hline JSK & $\begin{array}{l}\text { Jeunesse Sportive } \\
\text { de Kairouan }\end{array}$ & 1 & 1 & 2 & 2 & 2 & 27 & 35 \\
\hline SRS & Sfax Railway Sport & 1 & 0 & 0 & 3 & 5 & 25 & 34 \\
\hline
\end{tabular}

Sources : M. Kilani (2009) pour les données antérieures à 2008 et travaux de A. Langar en cours pour la période suivante et l'harmonisation.

\section{Hypothèses sur les mécanismes politiques et sportifs de transformation de la carte du football en Tunisie}

Le football n'est cependant pas un simple miroir des grandes inégalités spatiales en Tunisie entre littoral et intérieur ou entre métropoles et zones rurales. En raison de sa propre organisation à différents niveaux, il constitue également un acteur singulier de l'aménagement du territoire national. L'organisation des compétitions sportives est un sujet politique majeur pour un nouveau pays libéré de la domination coloniale. C'est pourquoi quelques jours seulement après la déclaration de l'Indépendance de la Tunisie, les responsables des clubs ont décidé de s'émanciper de la tutelle de la Fédération Française du Football en créant une association, la Fédération Tunisienne de Football, agréée le 29 mars 1957 par le visa nº 3017 (Kilani, 1998; Kilani, 2009). Au cours de ses premières années d'existence, le bureau fédéral de la FTF va tester différentes solutions pour remplir ses objectifs. Le plus important à retenir est l'existence d'une hiérarchie comportant cinq niveaux : Ligue nationale 1 ( $1^{\text {ère }}$ Division), Ligue nationale 2 ( $2^{\mathrm{e}}$ Division), Division d'honneur ( $3^{\mathrm{e}}$ Division), Ligue amateurs ( $4^{\mathrm{e}}$ Division), Ligues régionales ( $5^{\mathrm{e}}$ Division).

\section{Les régionalisations intermittentes de la deuxième division}

Le championnat de $2^{\mathrm{e}}$ Division a connu de nombreux remaniements entre 1956 et 2015. On assiste en effet à une alternance de périodes où ce championnat se compose d'une, deux, voire trois poules (respectivement période 1966-1970, la moitié des périodes et les années de 1983 à 1985). On assiste également à des modifications conséquentes du nombre d'équipes présentes et susceptibles d'accéder à l'élite: on passe ainsi d'un minimum de 10 équipes dans la période 1966-1970, à un maximum de plus de 40 équipes 
au moins une dans les années 1983-1985. Enfin, et ce n'est pas l'élément le moins important d'un point de vue symbolique, le nom des poules change au cours du temps reflétant l'évolution des représentations des grandes divisions territoriales du pays : la différenciation Nord-Sud, reprenant l'opposition historique du pays revient ainsi à trois reprises, tandis que la D2 demeure le constituant unique pour l'ensemble du territoire (Tableau 2).

Tableau 2 - Évolution du nombre de clubs et de poules en D2 entre 1956 et 2015

\begin{tabular}{|l|l|l|l|}
\hline Périodes & Nombre de poules & Nom des poules & Nombre de clubs par poule \\
\hline $1956-1965$ & 2 & Nord / Sud & 12 \\
\hline $1966-1970$ & 1 & Division 2 & 10 à 14 \\
\hline $1971-1974$ & 2 & Nord / Centre & 10 à 12 \\
\hline $1975-1979$ & 2 & Nord / Sud & 12 \\
\hline $1980-1982$ & 2 & Nord / Centre & 12 \\
\hline $1983-1985$ & 3 & Nord / Centre / Sud & 13 à 15 \\
\hline $1986-1994$ & 1 & Division 2 & 14 \\
\hline $1995-1998$ & 2 & Nord / Sud & 14 à 16 \\
\hline $1999-2011$ & 1 & Division 2 & 12 à 16 \\
\hline $2012-2015$ & 1 & Groupe A / Groupe B & 10 \\
\hline
\end{tabular}

Sources : M. Kilani (2009) pour les données antérieures à 2008 et travaux de A. Langar en cours pour la période suivante et l'harmonisation.

Vers la fin de la présidence de Bourguiba, on assiste durant une courte période de trois ans (1983-1985) à une multiplication du nombre de clubs de D2, à la faveur de la création de trois poules comptant 13 à 15 clubs chacune. Correspondant avec la période de préparation du Schéma National d'Aménagement du Territoire (Daoud, 2011), cette réorganisation temporaire de la géographie du football de D2 semble traduire une volonté politique de rééquilibrage au profit des territoires de l'intérieur et du Sud du pays (Bennasr, 2011 ; Daoud, 2011 ; Ben Jelloul, 2015). La distribution des clubs par poules en 1983 (illustration 8) met en évidence une grande région Sud mais aussi une montée en puissance de la région du Sahel qui se trouve réunifiée en une seule ligue alors qu'elle était antérieurement tiraillée entre Nord et Sud ou entre Nord et Centre. Cette période exceptionnelle a sans aucun doute favorisé l'accession temporaire de clubs à la Division 2 et peut-être par la suite à la Division 1 grâce à une triple action : accroissement du nombre de clubs de D2 favorisant mécaniquement l'entrée de nouveaux clubs de D3 en D2 entre 1982 et 1983; accroissement des chances de passage de D2 en D1 du fait de l'organisation en trois poules séparées; limitation des coûts de déplacement pour les clubs situés en périphérie du pays du fait du fractionnement spatial du championnat. 
Illustration 8 - Distribution des clubs et poules de football de Ligue 2 en Tunisie en 1983

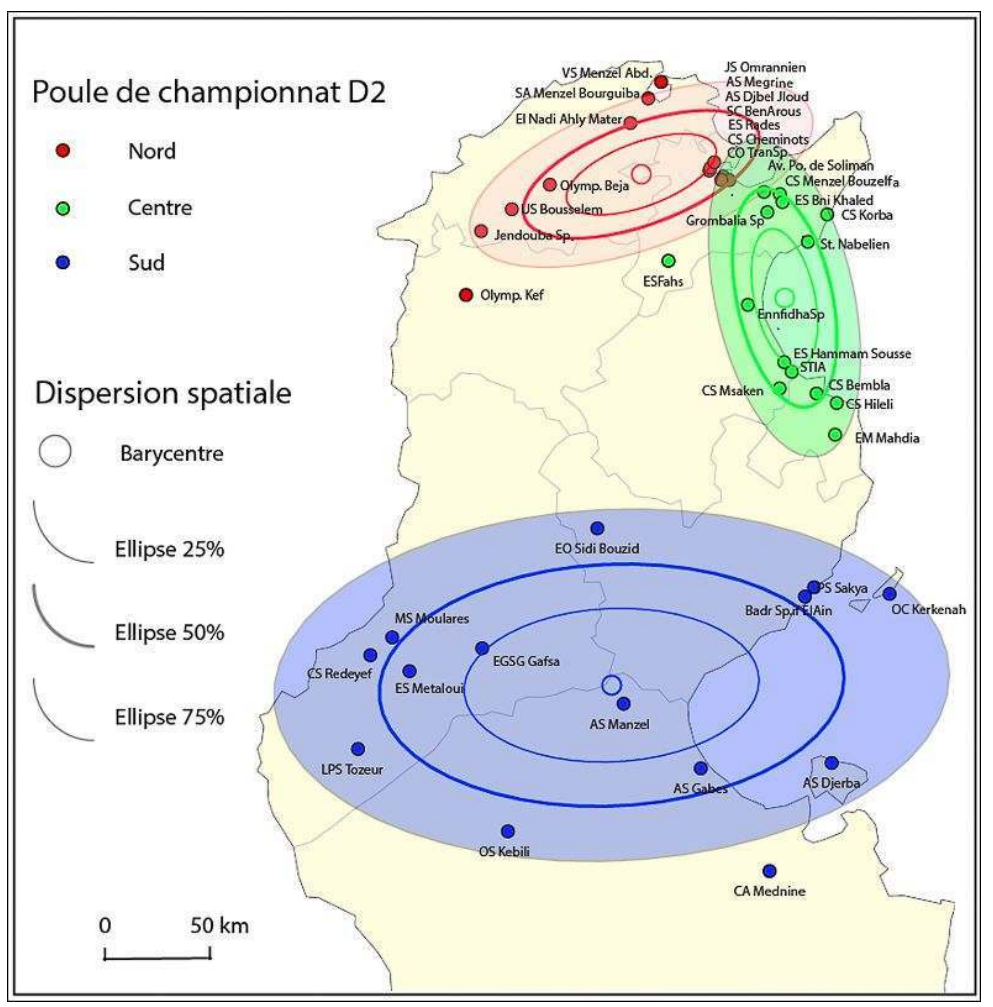

Sources : M. Kilani (2009) pour les données brutes et A. Langar pour l'harmonisation.

Mais naturellement les mécanismes vont jouer en sens contraire lors des périodes de réduction du nombre de poules et de clubs. En 1986, on revient à une poule unique de 14 clubs ce qui fait mécaniquement redescendre plus des deux-tiers des clubs en division inférieure.

\section{Ligues régionales et territorialités locales du football}

Une telle forme d'action volontariste en faveur du Sud conduit à se demander s'il existe un lien entre le projet politique et le projet sportif d'un pays. Plus précisément, on peut se demander si le tracé des ligues régionales de football relève d'objectifs d'équilibre territorial parallèles à ceux du pouvoir central en matière de pilotage et de régionalisation des compétences. Pour rompre avec l'héritage tribal et colonial, le gouvernement tunisien a en effet créé une organisation territoriale centralisée et découpée en trois niveaux : gouvernorats, délégations et secteurs. En 1957, la Tunisie est découpée en 14 gouvernorats et connaît ensuite des redécoupages administratifs successifs, chacun répondant à un objectif socio-économique bien déterminé (Ben Rebah, 2008).

Au cours de cette même année 1957, le bureau fédéral de la FTF choisit une organisation en régions beaucoup plus grandes puisqu'il délègue ses pouvoirs à quatre ligues régionales (Centre, Medjerda, Nord et Sud-Ouest) et les charge d'organiser les compétitions entre les clubs dans leurs régions (illustration 9). Il s'agit d'une forme de décentralisation où la FTF dirige directement les championnats de Tunisie des divisions Nationales et d'Honneur mais laisse les Ligues régionales organiser les compétitions au 
niveau local (Kilani, 2009). Ensuite, face à l'augmentation du nombre de clubs, les dirigeants de la FTF ont décidé d'augmenter le nombre de ligues, suivant en cela la même logique que les divisions administratives du pays qui voient également leur nombre augmenter avec la croissance de la population. En 1986, huit ligues régionales sont ainsi créées pour organiser les championnats régionaux de Divisions 3 et 4 : Nord, Tunis-Cap Bon, Nord-Ouest, Centre-Est, Centre, Sud, Sud-ouest, Sud-est. Après la révolution de 2011, le nombre de ces ligues passe à 12 avec l'ajout de quatre nouvelles Ligues: celle de Nabeul, Kairouan, Sidi Bouzid et Médenine. Au cours de la même période, le nombre de gouvernorats passe lui de 14 à 24 (illustration 9).

Illustration 9 - Gouvernorats et ligues régionales de football en Tunisie en 1957, 1986 et 2012

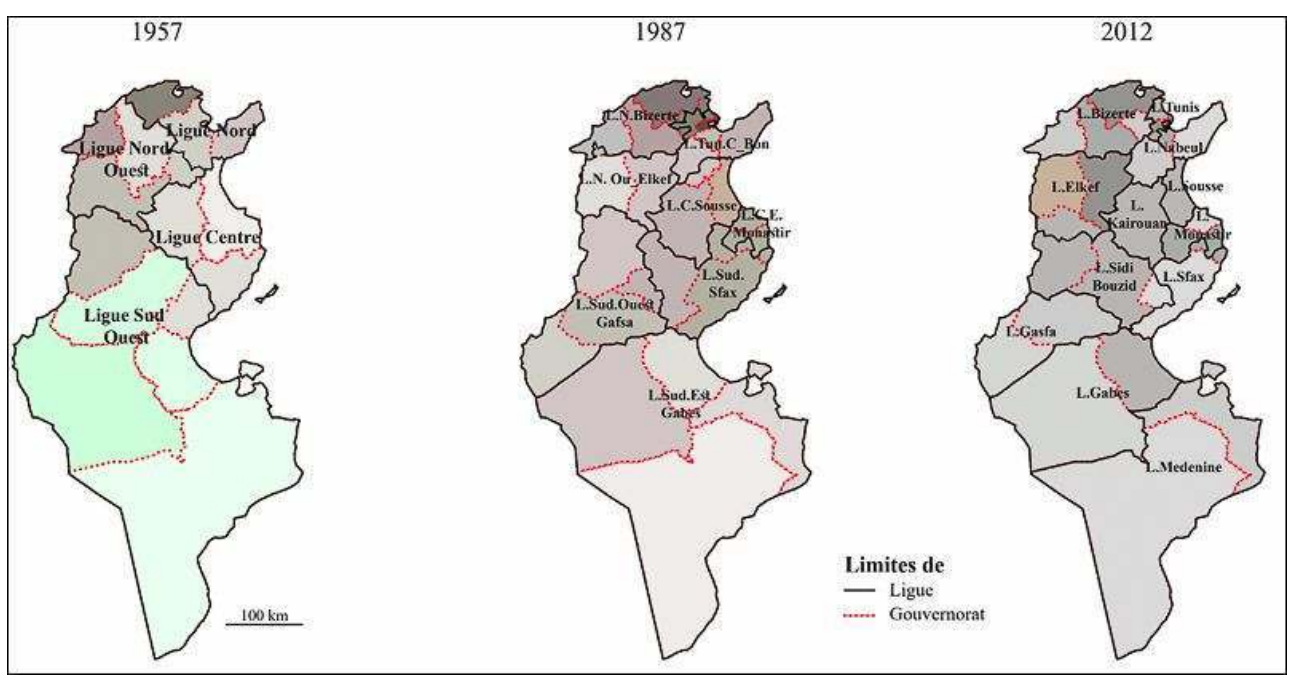

Source : Ministère de la Jeunesse et des sports (http : //www.mjs.tn/). Auteur : A. Langar.

On aurait pu imaginer que les gouvernements tunisiens successifs tentent de mettre en cohérence le maillage des ligues avec le maillage administratif. Or, quelle que soit la date retenue, il existe des discordances entre les deux. En 1957, la ligue du Sud-Ouest regroupe 6 des 14 gouvernorats existants mais coupe le gouvernorat de Sfax en deux : la partie Nord de ce gouvernorat est ainsi rattachée à la ligue du Centre. De même, en 1986, la ligue Sud-Sfax regroupe 3 gouvernorats: celui de Sfax, plus de la moitié du gouvernorat de Sidi-Bouzid et une partie du gouvernorat de Mahdia. En 2012, la ligue de Sidi-Bouzid fait partie des 4 ligues nouvellement créées et s'étend sur 3 gouvernorats : Sidi-Bouzid, la grande partie sud du gouvernorat de Kasserine et la délégation de Bir Ali ben Khalifa du gouvernorat de Sfax. On peut alors se demander dans quelle(s) mesure(s) les changements de découpage des ligues régionales suivent plutôt les performances des clubs de football.

Quand on s'intéresse à la situation de 1957, sur les 15 clubs de D1, aucune équipe n'appartient à la ligue de Sud-ouest. Et au vu de sa très grande superficie cet état de fait témoigne de la faible diffusion du football dans cette partie du pays. En 1987, on compte 8 ligues régionales, dont une pour le seul gouvernorat de Monastir, cher au Président Bourguiba. On constate une répartition beaucoup plus équilibrée des clubs de D1 entre celles-ci. C'est bien sûr dans la ligue de Tunis Cap Bon que l'on trouve le plus d'équipes de D1 (6 sur 14). Vient ensuite la ligue de Centre Sousse (3 équipes). À cette date, toutes les ligues régionales à l'exception de celle du Sud comptent au moins une équipe participant au championnat de D1. Après la révolution de 2011, le nombre de ligues régionales a encore augmenté, passant de 8 avant la révolution à 12 en 2014. On trouve toujours 
beaucoup d'équipes dans les principales villes de la zone littorale. Mais le fait marquant et inédit est la montée en puissance des équipes du Sud du pays qui représentent près de la moitié des clubs évoluant en D1 : 7 sur 16. En revanche, aucune équipe de D1 n'appartient aux deux ligues du Nord-Ouest - Le Kef et Sidi-Bouzid.

Ces observations suggèrent que le tracé des ligues régionales dépend moins des résultats des clubs participant au championnat national le plus prestigieux qu'à des principes d'accessibilité voire d'aménagement du territoire. La création d'une ligue semble en effet jouer un rôle d'impulsion et permettre en général la montée en puissance d'au moins un club jusqu'au niveau national. C'est ce que tend à montrer les créations des ligues de Sidi Bouzid et Médenine en 2012. Le tracé répond également à des considérations politiques (création de la ligue de Monastir) ou prend en compte la perception des espaces vécus par les acteurs, comme en témoignent les fluctuations des limites de la Ligue de Sfax. Interrogé en 2013 sur le rattachement de la délégation de Bir Ali Ben Klifa (délégation du gouvernorat de Sfax) à la ligue nouvellement créé de Sidi Bouzid, un dirigeant local ${ }^{6}$ expliquait que tout le monde n'est pas d'accord et qu'une partie des clubs de Bir Ali «se voient comme Sfaxiens pas Bouzidiens ». Pour les délégations de La Chebba et El Djem du gouvernorat de Mahdia, il invoquait alors le poids des héritages historiques: "Chebba et Djem ont appartenu avant à Sfax et aujourd'hui elles veulent continuer à jouer avec les clubs de Sfax ». Ce témoignage serait à confronter à celui des dirigeants des ligues voisines et à celui des premiers intéressés que sont les membres des clubs de football concernés par ces enjeux de tracé de limites moins anodins qu'il n'y parait (Grasland et al., 2015 ; Baron et al., 2017).

\section{Conclusion}

Au terme de cette analyse, il apparaît tout d'abord que la distribution spatiale des clubs de football tunisien d'élite que l'on a pu observer dans les années consécutives à la révolution de Jasmin (2012-2015) est beaucoup moins égalitaire qu'il n'y paraît puisqu'une poignée de clubs situés à Tunis et dans les métropoles littorales exerce un monopole certain sur les premières places du championnat depuis plus de 60 ans. Pour autant, la distribution spatiale des clubs de $1^{\text {ère }}$ et $2^{\mathrm{e}}$ Division s'est progressivement déconcentrée, à la fois vers l'intérieur mais aussi et surtout vers le Sud qui a clairement bénéficié d'une forte attention des pouvoirs politiques et sportifs, aussi bien à l'époque de Bourguiba qu'à celle de Ben Ali. Face à un héritage colonial qui avait concentré initialement le football à Tunis et dans quelques métropoles ou bassins industriels, les acteurs politiques et sportifs tunisiens ont fait preuve d'une très grande imagination en matière d'action sur l'espace et le territoire. Même s'il demeure difficile d'en évaluer les effets avec certitude, le découpage de la $2^{\mathrm{e}}$ Division en poules spatialement organisées tout comme le découpage des ligues régionales ont sans nul doute contribué à donner leur chance à de nombreux clubs des territoires périphériques du Sud et de l'intérieur.

31 De manière plus générale notre analyse confirme que l'histoire politique et celle du football en Tunisie sont étroitement liées. Dès son apparition, le football a été en permanence influencé par la situation politique du pays. Dans le même temps, il a été à plusieurs reprises utilisé par les acteurs politiques. Pour les uns, le football a pu être une vitrine et un moyen d'action pour lutter pour l'indépendance, ce qui a été le cas de plusieurs pays voisins à l'instar de l'Algérie (Abis et Ajmani, 2014 ; Kilani, 1998). 
Au-delà de la dimension historique du football en Tunisie et de son rôle dans le maintien mais aussi dans l'inflexion de certaines inégalités territoriales, des chantiers restent à explorer, notamment celui d'identifier ce qui se joue entre l'équipe nationale, les clubs de L1 et les clubs de supporters (Besson et Poli, 2007). Ce faisant, il conviendrait de dépasser le cadre national pour envisager les trajectoires de joueurs tunisiens du niveau local aux niveaux national et international pour mieux cerner la place de la Tunisie dans le marché des joueurs (Poli et Dietschy, 2006; Ravenel 1998-a ; Poli et Ravenel, 2005). Ce dernier point suppose également de travailler la question de la Tunisie comme territoire redistribuant les joueurs originaires de pays d'Afrique subsaharienne vers ses propres championnats mais aussi vers l'Europe ou encore les pays du Golfe (dans la lignée des travaux de Augustin, 2007; Gillon et al., 2010, Besson et al., 2010; Piraudeau, 2011; Lafabrègue et al., 2013).

\section{BIBLIOGRAPHIE}

Abis S, Ajmani D., 2014. Football et mondes arabes. Revue internationale et stratégique, $\mathrm{n}^{\circ} 94$, p. $143-150$.

Augustin J.-P., 1989. Les espaces des sports collectifs : l'exemple du département des Landes. Mappemonde, $\mathrm{n}^{\circ} 2$, p. 29-31.

Augustin J.-P., 1995. Sport, géographie et aménagement. Paris, Nathan, 256 p.

Augustin J.-P., 2007. Géographie du sport : spatialités contemporaines et mondialisation. Paris, Armand Colin, $224 \mathrm{p}$.

Baron M., de Ruffray S., Achouri W., Saadaoui K., Bennasr A., Grasland C., 2017. Complexité et enjeux des mailles territoriales. Variations à partir du cas tunisien. L'Année du Maghreb [En ligne], nº16, p. 109-126. http://journals.openedition.org/anneemaghreb/2985

Béguin H., 1979, Méthodes d'analyse géographique quantitative. Paris, LITEC, 252 p.

Belhedi A., 1994. L'inégal développement régional en Tunisie : Accumulation spatiale et littorisation. Cahiers de la Méditerranée, vol. 49, n 1, p. 133-156.

Belhedi A., 1999. Les disparités spatiales en Tunisie, état des lieux et enjeux. Méditerranée, vol 91, $\mathrm{n}^{\circ} 1$, p. 63-72.

Ben Jelloul M., 2015. Régionalisation et équité territoriale : vers une nouvelle gouvernance territoriale en Tunisie. Maghreb-Machrek, nº, p. 121-144.

Ben Rebah M., 2008. Cartographie dynamique et investigation territoriale : le cas de l'évolution du découpage administratif en Tunisie. Thèse de doctorat de géographie en co-tutelle internationale, Université Paris 7 et Université de Sfax, $334 \mathrm{p}$.

Bennasr A., Baron M., De Ruffray S., Grasland C., Guérin-Pace F., 2015. Dilemmes de la réforme régionale tunisienne. Revue d'Économie Régionale \& Urbaine, n5, p. 853-882.

Besson R., Poli R., Ravenel L., 2010. Comprendre les mécanismes des migrations «glo-balles» africaines Les trajectoires transnationales. Afrique contemporaine, $\mathrm{n}^{\circ}$ 233, p. 63-76. 
Besson R., Poli R., 2007. Des étrangers dans le stade ? Composition du public de Neuchâtel Xamax et interaction sociale. Bulletin de la Société Neuchâteloise de Géographie, n 50-51, p. 9-25.

Daoud A., 2011. La révolution tunisienne de janvier 2011 : une lecture par les déséquilibres du territoire. EchoGéo [En ligne], Rubrique Sur le Vif. URL : http://journals.openedition.org/ echogeo/12612 - DOI : 10.4000/echogeo.12612

Dietschy P., 2010. Histoire du football. Paris, Perrin, 619 p.

Drevon G., Gerber P., Klein O., Christophe E., 2014. Intégration spatiale des frontaliers à travers les activités quotidiennes. Le cas de la région métropolitaine luxembourgeoise. URBIA. Les Cahiers du développement urbain durable, $\mathrm{n}^{\circ} 16, \mathrm{p} .147-164$.

Gana A., 2011. Les inégalités socio-territoriales aux origines de la révolution tunisienne : défis du développement, enjeux pour la recherche. Lettre de l'IRMC, $\mathrm{n}^{\circ}$ 6, p. 18-20.

Gaubert V., 2016. Du football aux foot-ball : étude comparative de la géographie des cultures sportives « balle au pied ». Thèse de doctorat, Université Paris-Sorbonne - Paris 4.

Gillon P., Grosjean F., Ravenel L., Cassan D., Benoit-Guyod M., 2010. Atlas du sport mondial : business et spectacle : l'idéal sportif en jeu. Paris, Autrement, $80 \mathrm{p}$.

Gomez C., 2014. La diffusion du football en Amérique latine. Revue internationale et stratégique, $\mathrm{n}^{\circ} 2$, p. 151-159.

Grasland C., Baron M., Bennasr A., Brennetot A., de Ruffray S., Zanin C., 2015. Réformes régionales en France et en Tunisie. Éléments théoriques et méthodologiques pour cerner les territoires perçus. Maghreb-Machrek, n 4, p. 73-96

Grosjean F., 2004. Pour une approche spatialisée de la pratique sportive : l'exemple du football en milieu urbain. M@ppemonde [En ligne], n 76, 9 p. http://mappemonde.mgm.fr/num4/articles/ art04403.html

Keïta M., 2018. Typologie urbaine et accessibilité géographique potentielle des établissements de santé dits « modernes » dans le district de Bamako (Mali). Espace populations sociétés [En ligne], $\mathrm{n}$ ○ 1-2. URL : http://journals.openedition.org/eps/7707 - DOI : 10.4000/eps.7707

Kilani M., 2009. Guide-Foot saison 2008/2009. Tunis, Imp. Champs Elysées, 248 p.

Kilani M., 1998. Mémoire de football : l'histoire du football tunisien à travers les portraits de ses gloires. Tunis, Fédération Tunisienne de football.

Lafabrègue C., Tabé A., Helleu B, 2013. La fabrication des carrières migratoires des footballeurs africains. Le cas des joueurs béninois partis gagner leur vie en France. Revue européenne des migrations internationales, vol. $29, \mathrm{n}^{\circ} 1, \mathrm{p} .157-178$.

Lefebvre S., Roult R., Augustin J.-P. (dir.), 2013. Les nouvelles territorialités du sport dans la ville. Québec, PUQ, 236 p.

Piraudeau B., 2011. Les jeunes joueurs africains. Migrations Société, voL. 23, n 133, p. 11-29.

Poli, R., Dietschy, P., 2006. Le football africain entre immobilisme et extraversion. Politique africaine, $\mathrm{n}^{\circ} 2, \mathrm{p} .173-187$.

Poli, R., Ravenel, L., 2005. Les frontières de la «libre» circulation dans le football européen : vers une mondialisation des flux de joueurs? Espace populations sociétés. Space populations societies, (2005/2), p.293-303.

Ravenel L., 1996. La diffusion du football de haut niveau en France. Mappemonde, $\mathrm{n}^{\circ}$ 2, p. 14-18.

Ravenel L., 1998a. La géographie du football en France. Paris, PUF, 143 p. 
Ravenel L., 1998b. Hiérarchies urbaines, hiérarchies sportives : quand le football français s'écarte de la norme européenne. L'Espace géographique, vol. 27, n 4, p. 339-348.

Ravenel L., 2004. Pourquoi n'y a-t-il pas de rugby en Normandie? Un essai d'explication géographique. Norois. Environnement, aménagement, société, n 190, p. 85-95.

Saint-Julien T., 1982. Croissance industrielle et système urbain. Paris, Economica, 280 p.

Saint-Julien T., 1985. La diffusion spatiale des innovations. Montpellier, G.I.P. RECLUS, 48 p.

Sanders, L. (dir.), 2001. Modèles en analyse spatiale, Paris, Hermès - Lavoisier, 233 p.

Signoles P., 1978. Les inégalités régionales entre Tunis et la Tunisie. Revue française d'études politiques méditerranéennes, vol. 3, n 30-31, p. 42-59.

Taylor P.J., 1977. Quantitative Methods in Geography: an Introduction to Spatial Analysis. Boston, Houghton Mifflin Company, 386 p.

UNESCO, 1960. Rapport général de mission en Tunisie.

Zoubeidi M., 2007. 100 ans de football en Tunisie : ce que la mémoire aura gardé. Tunis-Ben Arous, Impr. Principale, $.251 \mathrm{p}$.

\section{ANNEXES}

Liste des clubs ayant passé au moins une année en Ligue 1 entre 1956 et 2015

\begin{tabular}{|l|l|l|l|}
\hline Code & Nom du club & Délégation (2014) & Nombre d'années en Ligue 1 \\
\hline AMS & AS Megrine & Megrine & 2 \\
\hline ASA & AS Ariana & L'Ariana Ville & 1 \\
\hline ASD & AS Djerba & Djerba Houmet Souk & 3 \\
\hline ASG & AS Gabes & Gabes Medina & 5 \\
\hline ASK & AS Kasserine & Kasserine Nord & 7 \\
\hline ASM & AS Marsa & La Marsa & 53 \\
\hline ASOE & AS Oued Elili & Oued Ellil & 2 \\
\hline CA & Club Africain & La Medina & 61 \\
\hline CAB & CA Bizerte & Bizerte Nord & 59 \\
\hline CM & CA Mednine & Medenine Sud & 3 \\
\hline COT & CO TranSp. & Sijoumi & 30 \\
\hline CSC & CS Cheminots & El Omrane Sup. & 9 \\
\hline CSHL & CS Hammam Lif & Hammam Lif & 55 \\
\hline
\end{tabular}




\begin{tabular}{|c|c|c|c|}
\hline CSK & CS Korba & Korba & 1 \\
\hline CSMB & CS Menzel Bouzelfa & Menzel Bouzelfa & 1 \\
\hline CSS & CS Sfaxien & Sfax Ville & 61 \\
\hline EGSG & EGS Gafsa & Gafsa Sud & 12 \\
\hline EMM & EM Mahdia & Mahdia & 9 \\
\hline ENAM & El Nadi Ahly Mater & Mateur & 4 \\
\hline EOGK & Etoile Goulette-Kram & La Goulette & 3 \\
\hline EOSB & EO Sidi Bouzid & Sidi Bouzid Est & 1 \\
\hline ESBK & ES Bni Khaled & Beni Khalled & 5 \\
\hline ESHS & ES Hammam Sousse & Hammam Sousse & 6 \\
\hline ESM & ES Metaloui & Metlaoui & 3 \\
\hline ESS & ES Sahel & Sousse Medina & 59 \\
\hline EST & ES Tunis & Bab Souika & 60 \\
\hline ESZ & ES Zarzis & Zarzis & 20 \\
\hline FCD & FC Djerissa & Djerissa & 1 \\
\hline GS & Grombalia Sp. & Grombalia & 2 \\
\hline JS & Jendouba Sp. & Jendouba & 3 \\
\hline JSK & JS Kairaounais & Kairouan Nord & 35 \\
\hline JSMet & JS Metouien & Bab Souika & 4 \\
\hline LPST & LPS Tozeur & Tozeur & 1 \\
\hline $\mathrm{OB}$ & Olymp. Beja & Beja Nord & 28 \\
\hline OCK & OC Kerkenah & Kerkenah & 13 \\
\hline OK & Olymp. Kef & Kef Est & 13 \\
\hline PFCB & PFC Bizerte & Bizerte Sud & 3 \\
\hline PS & Patr. Sousse & Sousse Medina & 2 \\
\hline SAMB & SA Menzel Bourguiba & Menzel Bourguiba & 4 \\
\hline SG & St. Gabsien & Gabes Medina & 12 \\
\hline
\end{tabular}




\begin{tabular}{|l|l|l|l|}
\hline SP & St. Populaire & Zahra & 4 \\
\hline SRS & Sfax Railway Sp. & Sfax Ville & 34 \\
\hline SS & St. Soussien & Sousse Medina & 12 \\
\hline SSS & St. Sp.if Sfaxien & Sfax Sud & 7 \\
\hline ST & St. Tunisien & Le Bardo & 61 \\
\hline STIA & STIA & Sousse Riadh & 1 \\
\hline USBG & US Ben Guerden & Ben Guerden & 1 \\
\hline USMA & US Magrebin & Tunis & 4 \\
\hline USMF & US Menzel Feress & Moknine & 1 \\
\hline USMO & US Monastir & Monastir & 43 \\
\hline UST & US Tunisien & Bab El Bhar & 10 \\
\hline
\end{tabular}

\section{NOTES}

1. http://www.cahiersdufootball.net/article-le-seul-endroit-ou-la-police-n-etait-plus-toutepuissante-c-etait-le-virage-5162 (consulté le 18 juillet 2018).

2. Extrait de la page internet "Le sport à Menzel : une si longue histoire» http:// menzelbourguiba-ex-ferryville.over-blog.fr/article-le-sport-a-menzel-une-si-longue-

histoire-64057430.html (consulté le 18 juillet 2018).

3. Base de données concernant les équipes présentes au moins une fois dans les championnats de Ligues 1 et 2 depuis 1956, localisées géographiquement et caractérisées par la place occupée dans les championnats entre 1956 et 2012. Les changements de nom qu'elles ont pu connaître ont également été enregistrés.

4. La distance-type a souvent été utilisée en géographie pour rendre compte de la dispersion spatiale du peuplement (Béguin, 1971 et 1979 ; Taylor, 1977) ou des activités économiques (SaintJulien, 1982). La distance-type joue un rôle analogue à celui des ellipses de dispersion dans la mesure où elle permet de définir un cercle à l'intérieur duquel on doit trouver les deux tiers des équipes de football.

5. Une classification des profils des clubs ayant circulé entre les deux divisions dépasse le cadre $\mathrm{du}$ présent article. Néanmoins, nous avons fourni en annexe de cet article, à titre indicatif, un tableau du nombre de saisons passées en D1 par les clubs avec leur nom et leur sigle (Annexe 1).

6. Dont l'anonymat est préservé à sa demande. 


\section{RÉSUMÉS}

En préparation de la coupe du monde de football en Russie aux mois de juin et juillet 2018, un article publié dans le journal Le Monde au mois de mars soulignait que les Tunisiens semblaient moins attachés à leur équipe nationale qu'aux principaux clubs évoluant dans le championnat de première division. Ce constat est à mettre en regard avec la manière dont ce sport s'est implanté et diffusé durant le protectorat français, le rôle politique qu'il a pu jouer que ce soit dans la marche vers l'indépendance ou, plus récemment, lors des grandes manifestations de la révolution de jasmin durant l'hiver 2010-2011. Enfin, le football, premier sport en Tunisie par le nombre de licenciés, s'adosse mais aussi contribue en partie à entretenir les grandes inégalités territoriales entre le littoral et l'intérieur, le Nord et le Sud par l'implantation des principales équipes participant au championnat le plus prestigieux, celui de la Ligue ou Division 1. Toutefois, quand sont prises en compte les Ligues régionales, force est de constater que ce sport joue aussi un rôle notable dans les manières dont l'aménagement et les rééquilibrages au sein du territoire national peuvent être appréhendés.

Before the Soccer World Cup in Russia in June and July 2018, the newspaper Le Monde underlined in March that Tunisians seemed less attached to their national team than to the main clubs evolving in the championship of first division. This leads to be interested in the way this sport became established and spread during the French protectorate, the political role which it played whether it is in the walking towards the independence or, more recently, during the big demonstrations of the revolution of jasmine during winter 2010-2011. Finally, the soccer, the first sport in Tunisia by the number of graduates, leans but also contributes partially to maintain main territorial disparities between the coast and the inside, the North and the South by the setting-up of the main teams participating in the most prestigious championship, that of the First League. However, when are taken into account the regional Leagues, we have to admit that this sport also plays a notable role in the manners among which the arrangement and the rebalancings within the national territory can be arrested.

\section{INDEX}

Keywords : soccer, Tunisia, planning, evolution, hierarchy, league

Mots-clés : football, Tunisie, aménagement, évolution, hiérarchie, ligue

\section{AUTEURS}

\section{ALI LANGAR}

Ali Langar, alilangar2@gmail.com, est doctorant en géographie et rattaché à l'Université Paris Diderot, UMR Géographie-cités CNRS 8504, et à l'Université de Sfax, Laboratoire Syfacte. Il a récemment publié :

- Langar A., Baron M., 2017. Cartographier le football sur le territoire tunisien. Quels objets ? Pour 
quels phénomènes ? In Dhieb M. (dir.), L'information géographique et le monde changeant, Cahiers du C.E.R.E.S., Série Cartographie, n³, p. 249-261.

- Langar A., 2016, Le football en Tunisie : enjeux politiques et maillages. In Hadrossek C et al. (dir.), Maillages territoriaux, Démocratie et Élection, recueil de proceedings du colloque international de Monastir [En ligne], p. 47-54. https://halshs.archives-ouvertes.fr/hal-01383180v1

- Langar A., 2016. Football et hiérarchie urbaine en Tunisie (1955-2015) [Pster]. In Hadrossek C. et al. (dir.), Maillages territoriaux, Démocratie et Élection, recueil de proceedings du colloque international de Monastir [En ligne], p.239-246. https://halshs.archives-ouvertes.fr/ hal-01383180v1

\section{MYRIAM BARON}

Myriam Baron, myriam.baron@u-pec.fr, est Professeure de Géographie à l'Université Paris Est Créteil et membre de Lab'Urba EA 3482. Elle a récemment publié :

- Nader B., Pandato L., Mobillon V., Bochaton A., Charreire H., Baron M., 2018. Vieillissement de la population et ville durable : quels enjeux ? Revue Pollution Atmosphérique, n²37-238, $12 \mathrm{p}$.

- Langar A., Baron M., 2017. Cartographier le football sur le territoire tunisien. Quels objets ? Pour quels phénomènes? In Dhieb M. (dir.), L'information géographique et le monde changeant, Cahiers du C.E.R.E.S., Série Cartographie, n³, p. 249-261.

- Baron M., De Ruffray S., Achouri W., Saadaoui K., Bennasr A., Grasland C., 2017. Complexité et enjeux des mailles territoriales. Variations à partir du cas tunisien. L'Année du Maghreb [En ligne], n¹6, p. 109-126. http://journals.openedition.org/anneemaghreb/2985

\section{CLAUDE GRASLAND}

Claude Grasland, claude.grasland@parisgeo.cnrs.fr, est Professeur de Géographie à l'Université Paris 7 Paris-Diderot et membre de l'UMR Géographie-cités, CNRS 8504. Il a récemment publié : - Baron M., De Ruffray S., Achouri W., Saadaoui K., Bennasr A., Grasland C., 2017. Complexité et enjeux des mailles territoriales. Variations à partir du cas tunisien. L'Année du Maghreb [En ligne], n¹6, p. 109-126. http://journals.openedition.org/anneemaghreb/2985

- Grasland C., Lamarche-Perrin R., Loveluck B., Pecout H., 2016. L'agenda géomédiatique international: analyse multidimensionnelle des flux d'actualité. L'Espace géographique, vol. 45, n - 1, p. 25-43.

- Bennasr A., Baron M., De Ruffray S., Grasland C., Guerin-Pace F., 2015. Dilemmes de la réforme régionale tunisienne. Revue d'Économie Régionale \& Urbaine, n 5, p. 853-882. 